\title{
Entangled Webs: Tight Bound for Symmetric Sharing of Entanglement
}

\author{
Masato Koashi ${ }^{1}$, Vladimír Bužek ${ }^{1,2}$, and Nobuyuki Imoto ${ }^{1}$ \\ ${ }^{1}$ CREST Research Team for Interacting Carrier Electronics, School of Advanced Sciences, \\ The Graduate University for Advanced Studies (SOKEN), Hayama, Kanagawa, 240-0193, Japan \\ ${ }^{2}$ Institute of Physics, Slovak Academy of Sciences, Dúbravská cesta 9, 84228 Bratislava, Slovakia

\begin{abstract}
Quantum entanglement cannot be unlimitedly shared among arbitrary number of qubits. Larger the number of entangled pairs in an $N$-qubit system, smaller the degree of bi-partite entanglement is. We analyze a system of $N$ qubits in which an arbitrary pair of particles is entangled. We show that the maximum degree of entanglement (measured in the concurrence) between any pair of qubits is $2 / N$. This tight bound can be achieved when the qubits are prepared in a pure symmetric (with respect to permutations) state with just one qubit in the basis state $|0\rangle$ and the others in the basis state $|1\rangle$.
\end{abstract}

Schrödinger [1] has identified quantum entanglement as the key ingredient in the paradigm of quantum mechanics. Throughout the whole history of modern quantum mechanics the mystery of quantum entanglement puzzled generations of physicists [2]. On the other hand in the last decade the entanglement has been recognized as an important resource for quantum information processing. In particular, quantum computation [3], quantum teleportation [4], quantum dense coding [5], certain types of quantum key distributions [6] and quantum secret sharing protocols [7], are rooted in the existence of quantum entanglement.

In spite of all the progress in the understanding of the nature of quantum entanglement there are still open questions which have to be answered. In particular, it is not clear yet how to quantify uniquely the degree of entanglement 8 12], or how to specify the inseparability conditions for bi-partite multi-level systems (qudits) [13. Further problem which waits for a thorough illumination is the multiparticle entanglement [14]. There are several aspects of quantum multiparticle correlations. For instance, it is the investigation of intrinsic $n$-party entanglement (i.e, generalizations of the GHZ state [15]). Another aspect of the multiparticle entanglement is that in contrast to classical correlation it cannot freely be shared among many objects. In particular, Coffman et al. [16] have studied a set of three qubits $A, B$ and $C$. It has been shown that the sum of the entanglement (measured in terms of the tangle [11]) between the particles $A B$ and the particles $A C$ is smaller or equal to the entanglement between the particle $A$ and the subsystem $B C$. Wootters [17] has considered an infinite collection of qubits arranged in an open line, such that every pair of nearest neighbors is entangled. In this translationally invariant entangled chain the maximal closest-neighbor (bi-partite) entanglement (measured in the concurrence) is bounded by the value $1 / \sqrt{2}$ (it is not known whether this bound is achievable) [17.

In this Letter, we consider a finite system of $N$ qubits in which each pair out of $N(N-1) / 2$ possible pairs is entangled. We show that the maximal possible bi-partite concurrence in this case is equal to $2 / N$. The derivation of this tight bound on the concurrence is the main result of our Letter.

The problem is formally posed as follows. Suppose that $N$ qubits, indexed by $l=1,2, \ldots, N$, are in the state $\hat{\rho}_{N}$, and choose a basis $\{|1\rangle,|0\rangle\}$ for each qubit. We require that the matrix form of the marginal density operator $\rho_{l l^{\prime}}$ for a pair of qubits $l$ and $l^{\prime}$, represented in the chosen basis, is independent of the choice of $l$ and $l^{\prime}$. Note that this requirement is satisfied if $\hat{\rho}_{N}$ is invariant under any permutation of qubits. The question is to find the maximum degree of entanglement between a pair of qubits.

It is convenient to suppose that each qubit is a spin$1 / 2$ particle with the spin operator $\hat{\boldsymbol{s}}^{(l)}(l=1, \ldots N)$. The Hilbert space of the subsystem composed of qubits 1 and 2 is a direct sum of the subspaces for the total spin 0 and 1 , with the projectors $\hat{P}_{0}$ and $\hat{P}_{1}$ onto each subspace, respectively. Under the condition $\hat{\rho}_{12}=\hat{\rho}_{21}(\equiv \hat{\rho})$, we have $\hat{P}_{0} \hat{\rho} \hat{P}_{1}=\hat{P}_{1} \hat{\rho} \hat{P}_{0}=0$, since these operators change their signs under the permutation of the two qubits. Let us define irreducible tensors $\hat{T}_{j, q}^{(k)}$ of rank $k=0,1,2$ and components $q$ such that $\hat{T}_{j, q}^{(k)} \equiv \sum_{m, m^{\prime}}\left\langle k, q \mid 1, m ; 1, m^{\prime}\right\rangle\left(\sum_{l=1}^{j} \hat{s}_{m}^{(l)}\right)\left(\sum_{l^{\prime}=1}^{j} \hat{s}_{m^{\prime}}^{\left(l^{\prime}\right)}\right)$, where $\hat{s}_{ \pm 1}^{(l)} \equiv \mp\left(\hat{s}_{x}^{(l)} \pm i \hat{s}_{y}^{(l)}\right) / \sqrt{2}, \quad \hat{s}_{0}^{(l)} \equiv \hat{s}_{z}^{(l)}$, and $\left\langle k, q \mid 1, m ; 1, m^{\prime}\right\rangle$ is the Clebsh-Gordon coefficient for forming a total spin $k$ state from two spin-1 particles. The spin-1 part of the density operator $\hat{\rho}$ can be expanded by $\hat{T}_{2, q}^{(k)}$ as $\hat{P}_{1} \hat{\rho} \hat{P}_{1}=\sum_{k, q} \alpha_{k, q} \hat{T}_{2, q}^{(k)}$, and the coefficients $\alpha_{k, q}$ are obtained by the relation $\alpha_{k, q} \operatorname{Tr}\left(\hat{T}_{2,-q}^{(k)} \hat{T}_{2, q}^{(k)}\right)=$ $\operatorname{Tr}\left(\hat{T}_{2,-q}^{(k)} \hat{\rho}\right)=\left\langle\hat{T}_{2,-q}^{(k)}\right\rangle$, where we denote $\operatorname{Tr}\left(\ldots \hat{\rho}_{N}\right)$ as $\langle\ldots\rangle$. From the symmetry of $\hat{\rho}_{N}$, we have $\left\langle\hat{T}_{N, q}^{(1)}\right\rangle=$ $(N / 2)\left\langle\hat{T}_{2, q}^{(1)}\right\rangle$ and $\left\langle\hat{T}_{N, q}^{(2)}\right\rangle=(N(N-1) / 2)\left\langle\hat{T}_{2, q}^{(2)}\right\rangle$. With $\hat{\rho}_{N}$ given, it is convenient to choose the $x, y$, and $z$ axis as the principal axes for the tensor of the second-order correlation for the total spin $\hat{\boldsymbol{S}} \equiv \sum_{l=1}^{N} \hat{\boldsymbol{s}}^{(l)}$ of the $N$ qubits, namely, $\left\langle\hat{S}_{\mu} \hat{S}_{\nu}+\hat{S}_{\nu} \hat{S}_{\mu}\right\rangle=2 S_{\mu}^{2} \delta_{\mu \nu}$ with $S_{\mu}^{2} \equiv\left\langle\hat{S}_{\mu}^{2}\right\rangle$ (here and henceforth, suffices $\mu$ and $\nu$ represent $x, y, z$ ). Matrix elements for $\hat{\rho}$ then takes a simple form on the basis $\{|\uparrow \uparrow\rangle-|\downarrow \downarrow\rangle,|\uparrow \uparrow\rangle+|\downarrow \downarrow\rangle,|\uparrow \downarrow\rangle+|\uparrow \downarrow\rangle,|\uparrow \downarrow\rangle-|\uparrow \downarrow\rangle\}$ as follows, 


$$
\rho=\frac{1}{N}\left(\begin{array}{cccc}
A_{x} & \left\langle\hat{S}_{z}\right\rangle & i\left\langle\hat{S}_{y}\right\rangle & 0 \\
\left\langle\hat{S}_{z}\right\rangle & A_{y} & \left\langle\hat{S}_{x}\right\rangle & 0 \\
-i\left\langle\hat{S}_{y}\right\rangle & \left\langle\hat{S}_{x}\right\rangle & A_{z} & 0 \\
0 & 0 & 0 & A_{0}
\end{array}\right)
$$

where we have introduced nonnegative parameters $A_{0} \equiv$ $\frac{N(N+2)-4\left\langle\hat{\boldsymbol{S}}^{2}\right\rangle}{4(N-1)}$ and $A_{\mu} \equiv \frac{N^{2}-4 S_{\mu}^{2}}{2(N-1)}-A_{0}$, that satisfy $A_{x}+A_{y}+A_{z}+A_{0}=N$.

As a measure of entanglement between the two qubits, we use the 'concurrence' introduced by Hill and Wootters [11]. The concurrence $C$ can be calculated as follows. Let $\tilde{\rho}$ be the time reversal of $\rho$, which is obtained by changing the sign of spin $\langle\hat{\boldsymbol{S}}\rangle$ in the expression (1). The eigenvalues of $\rho \tilde{\rho}$ are all real and non-negative, and let the square roots of those be $l_{1}, l_{2}, l_{3}$, and $l_{4}$ in decreasing order. The concurrence $C$ is then given by $C=\max \left\{l_{1}-l_{2}-l_{3}-l_{4}, 0\right\}$. In the present case, one of the eigenvalues of $\rho \tilde{\rho}$ is $\left(A_{0} / N\right)^{2}$. Let us denote the other three as $\left(\lambda_{1} / N\right)^{2},\left(\lambda_{2} / N\right)^{2}$, and $\left(\lambda_{3} / N\right)^{2}$ in decreasing order, and introduce parameters $\beta \equiv \lambda_{1}+\lambda_{2}+\lambda_{3}$ and $\gamma \equiv \lambda_{1}-\lambda_{2}-\lambda_{3}$. The concurrence is then given by $C=\max \left\{\left(\gamma-A_{0}\right) / N,\left(A_{0}-\beta\right) / N, 0\right\}$, where allowances are made for the possible order of $A_{0}$ and $\lambda_{1}$.

In the following, we first fix the parameters $S_{\mu}^{2}$ (hence $A_{\mu}$ and $\left.A_{0}\right)$, and maximize $\gamma$ with respect to $(X, Y, Z) \equiv$ $\left(\left\langle\hat{S}_{x}\right\rangle^{2},\left\langle\hat{S}_{y}\right\rangle^{2},\left\langle\hat{S}_{z}\right\rangle^{2}\right)$. We then move $S_{i}^{2}$ to obtain the global maximum of $\left(\gamma-A_{0}\right) / N$, that turns out to be, as we shall see, the maximum of the concurrence. For simplicity, we assume that $S_{z}^{2}>S_{y}^{2}>S_{x}^{2}$ (hence $A_{z}<$ $\left.A_{y}<A_{x}\right)$. The states with some parameters equal will be considered as the limiting cases.

There are two simple bounds for the allowed values of $(X, Y, Z)$. One is a necessary condition for $\rho$ to be physical. The eigenvalues for $\rho$ must be nonnegative, and the boundary is given by the surface that satisfies $\operatorname{det}(\rho)=0$. Calculating from (11), this surface turns out to be a plane, and the condition for $(X, Y, Z)$ is

$$
f_{A} \equiv A_{x} A_{y} A_{z}-A_{x} X-A_{y} Y-A_{z} Z \geq 0 .
$$

The other one is a requirement necessary for the spin correlations. From the inequality $\left\langle\left[\sum_{i}\left\langle\hat{S}_{i}\right\rangle\left(\hat{S}_{i}-\right.\right.\right.$ $\left.\left.\left.\left\langle\hat{S}_{i}\right\rangle\right) / S_{i}^{2}\right]^{2}\right\rangle \geq 0$, we obtain

$$
f_{S} \equiv 1-\frac{X}{S_{x}^{2}}-\frac{Y}{S_{y}^{2}}-\frac{Z}{S_{z}^{2}} \geq 0 .
$$

Any physical state thus falls in the region $V$, that is defined by $f_{A} \geq 0, f_{S} \geq 0$, and $X, Y, Z \geq 0$

The relations that connect $(X, Y, Z)$ and $\lambda_{i}$ are obtained by expanding $\operatorname{det}\left[\rho \tilde{\rho}-\left(\lambda^{2} / N^{2}\right) I\right]=\prod_{i}\left(\lambda_{i}^{2}-\lambda^{2}\right)$ and equating the coefficients of $\lambda^{m}$. There are three independent equations, and it is convenient to take the following set:

$$
\begin{aligned}
f_{0} & \equiv A_{x}^{2}+A_{y}^{2}+A_{z}^{2}-2 X-2 Y-2 Z \\
& =\lambda_{1}^{2}+\lambda_{2}^{2}+\lambda_{3}^{2}
\end{aligned}
$$

$$
\begin{aligned}
f_{A} & =\lambda_{1} \lambda_{2} \lambda_{3} \\
f_{B} & \equiv-B_{x} B_{y} B_{z}\left(B_{x}+B_{y}+B_{z}-\frac{4 X}{B_{x}}-\frac{4 Y}{B_{y}}-\frac{4 Z}{B_{z}}\right) \\
& =\lambda_{1}^{4}+\lambda_{2}^{4}+\lambda_{3}^{4}-2\left(\lambda_{1}^{2} \lambda_{2}^{2}+\lambda_{2}^{2} \lambda_{3}^{2}+\lambda_{3}^{2} \lambda_{1}^{2}\right),
\end{aligned}
$$

where $B_{\mu} \equiv A_{x}+A_{y}+A_{z}-2 A_{\mu}$. The relation $A_{x}>A_{y}>$ $A_{z}>0$ implies that $B_{z}>B_{y}>\left|B_{x}\right|$. Note that the sign of $f_{B}$ is the same as that of $\gamma$, since we can factorize as $f_{B}=\beta \gamma\left(\lambda_{1}+\lambda_{3}-\lambda_{2}\right)\left(\lambda_{1}+\lambda_{2}-\lambda_{3}\right)$.

Let $W$ be the region defined by $f_{A} \geq 0$ and $f_{B} \geq 0$. As a function of $(X, Y, Z), \gamma$ is continuous in the region $W$ including the boundaries. The gradient $\nabla \gamma \equiv$ $(\partial \gamma / \partial X, \partial \gamma / \partial Y, \partial \gamma / \partial Z)$ can formally be obtained by using the three relations (四). The result is

$$
\frac{\partial \gamma}{\partial X}=\frac{\left(\gamma+B_{y}\right)\left(\gamma+B_{z}\right)}{\kappa},
$$

where $\kappa \equiv 2\left(\lambda_{1}-\lambda_{2}\right)\left(\lambda_{1}-\lambda_{3}\right)\left(\lambda_{2}+\lambda_{3}\right) \geq 0$, and the other two are obtained by the cyclic exchange. At the inner points of $W, \lambda_{1}>\lambda_{2}$ since $\gamma>0$, and $\lambda_{3}>0$ since $f_{A}>0$. The parameter $\kappa$ is hence positive, and the gradient $\nabla \gamma$ exists. Since $B_{z}>B_{y}>0$, we have $\partial \gamma / \partial X>0$ for the inner points of $W$.

The geometry of $W$ and $V$ is derived as follows. Let the points on which the planes $f_{B}=0, f_{A}=0$, and $f_{S}=0$ intersect $X$-axis be $P_{B X}, P_{A X}$, and $P_{S X}$, respectively, and denote other six points on $Y$ - and $Z$-axis similarly. The relation $A_{x} A_{y}-\left(B_{x}+B_{y}+B_{z}\right) B_{z} / 4=$ $B_{x} B_{y} / 4$, and the ones obtained by the cyclic exchange of $\{x, y, z\}$, tells us the following. When $B_{x}>0$, the triangle $\pi_{B} \equiv P_{B X} P_{B Y} P_{B Z}$ does not intersect with $\pi_{A} \equiv P_{A X} P_{A Y} P_{A Z}$, and lies closer to the origin. $W$ is the sandwiched region of the two triangles. The triangle $\pi_{S} \equiv P_{S X} P_{S Y} P_{S Z}$ may intersect with $\pi_{A}$ and $\pi_{B}$ or not. When $B_{x}<0, f_{B}>0$ (hence $\gamma>0$ ) is satisfied by all the points that satisfy $f_{A} \geq 0$. When $B_{x}=0, \gamma=0$ for the points on $Y Z$-plane, and $\gamma>0$ for $X>0$. Combining these observation with $\partial \gamma / \partial X>0$, we conclude that in $V, \gamma$ takes its maximum on points on the boundaries $\pi_{A}$ or $\pi_{S}$, and never on the inner points.

To determine the behavior of $\gamma$ on the boundaries, we first derive the value of $\gamma$ on the axes explicitly. For the points on $Z$-axis and satisfy (2), the roots $\left\{\lambda_{i}\right\}$ are found to be $\left\{A_{z},\left[\sqrt{\left(A_{x}+A_{y}\right)^{2}-4\left\langle\hat{S}_{z}\right\rangle^{2}} \pm\left(A_{x}-A_{y}\right)\right] / 2\right\}$. The expression for $\gamma$ depends on which is the largest root. The roots for the points on the other two axes are similarly obtained. Applying these for the vertices of $\pi_{A}$, we have $\gamma\left(P_{A X}\right)=B_{y}$ and $\gamma\left(P_{A Y}\right)=\gamma\left(P_{A Z}\right)=\left|B_{x}\right|$. On the vertices of $\pi_{S}, \gamma$ is well-defined only when they are in the region $W$. When $P_{S Z}$ is in $W$,

$$
\begin{aligned}
0 & \leq 4\left(A_{x} A_{y}-S_{z}^{2}\right) \\
& =\left(A_{z}+A_{0}\right)\left(A_{z}+A_{0}-2\right)-\left(A_{x}-A_{y}\right)^{2},
\end{aligned}
$$

and hence $A_{z}>2-A_{0}$. Then $\gamma$ is written as 


$$
\begin{aligned}
\gamma\left(P_{S Z}\right) & =A_{z}-\sqrt{\left(A_{x}+A_{y}\right)^{2}-4 S_{z}^{2}} \\
& =A_{z}-\sqrt{\left(A_{z}+A_{0}\right)\left(A_{z}+A_{0}-2\right)}
\end{aligned}
$$

This is a decreasing function of $A_{z}$ and noting $S_{z}^{2}>0$, we have $2-A_{0}>\gamma\left(P_{S Z}\right)>N /(N-1)-A_{0} \equiv \gamma_{m}$. Similarly, $\gamma\left(P_{S X}\right)>\gamma_{m}$ and $\gamma\left(P_{S Y}\right)>\gamma_{m}$ if those points are in $W$.

Next, we will evaluate the gradient on the boundaries. On the boundary $\pi_{A}, \kappa$ is not always positive, but $\kappa=0$ is possible only for the following two cases. (a) $\lambda_{1}=\lambda_{2}$. This occurs only when $f_{A}=f_{B}=0$, or equivalently, on the segment $P_{A Y} P_{A Z}$ when $B_{x}=0$. (b) $\lambda_{2}=\lambda_{3}=0$. This occurs if and only if $f_{A}=0$ and $\left(f_{0}\right)^{2}-f_{B}=0$. The intersection of $f_{A}=0$ and $\left(f_{0}\right)^{2}-f_{B}=0$ is a parabola. The point $P_{0}\left(A_{z}^{2}\left(A_{x}-\right.\right.$ $\left.\left.A_{y}\right) /\left(A_{x}-A_{z}\right), 0, A_{x}^{2}\left(A_{y}-A_{z}\right) /\left(A_{x}-A_{z}\right)\right)$ is on this parabola. At $P_{0}, \boldsymbol{u}=\left(A_{x}, A_{y}, A_{z}\right)$ is normal to $f_{A}=0$, and $\boldsymbol{v}=\left(-A_{x} B_{y}, A_{y} B_{y}-2 A_{x} A_{z},-A_{z} B_{y}\right)$ is normal to $\left(f_{0}\right)^{2}-f_{B}=0$. Since $\boldsymbol{u} \times \boldsymbol{v}$ has a vanishing $Y$ component, the parabola is tangent to $X Z$-plane at $P_{0}$. Another point $\left(A_{y}^{2}\left(A_{x}-A_{z}\right) /\left(A_{x}-A_{y}\right),-A_{x}^{2}\left(A_{y}-A_{z}\right) /\left(A_{x}-\right.\right.$ $\left.\left.A_{y}\right), 0\right)$, on which $Y<0$, is also on the parabola. We thus conclude that the gradient exists on $\pi_{A}$ except for the segment $P_{A Y} P_{A Z}$ and $P_{0}$.

Let us define three particular directions on $\pi_{A}$ as $\boldsymbol{q}^{y x} \equiv\left(A_{y} A_{z},-A_{z} A_{x}, 0\right), \boldsymbol{q}^{z x} \equiv\left(A_{y} A_{z}, 0,-A_{x} A_{y}\right)$, and $\boldsymbol{q}^{y z} \equiv\left(0,-A_{z} A_{x}, A_{x} A_{y}\right)$. The differential coefficients of $\gamma$ for these directions are calculated to be $\boldsymbol{q}^{y x} \cdot \boldsymbol{\nabla} \gamma=$ $A_{z}\left(B_{z}^{2}-\gamma^{2}\right)\left(A_{x}-A_{y}\right) / \kappa, \boldsymbol{q}^{z x} \cdot \nabla \gamma=A_{y}\left(B_{y}^{2}-\gamma^{2}\right)\left(A_{x}-\right.$ $\left.A_{z}\right) / \kappa$, and $\boldsymbol{q}^{y z} \cdot \nabla \gamma=A_{x}\left(\gamma^{2}-B_{x}^{2}\right)\left(A_{y}-A_{z}\right) / \kappa$. Noting that $\gamma\left(P_{A X}\right)=B_{y}$ and $\gamma\left(P_{A Y}\right)=\gamma\left(P_{A Z}\right)=\left|B_{x}\right|$, we conclude that $\boldsymbol{q}^{y x} \cdot \nabla \gamma>0, \boldsymbol{q}^{z x} \cdot \nabla \gamma>0$ and $\boldsymbol{q}^{y z} \cdot \nabla \gamma>0$ on $\pi_{A}$ except for on the segment $P_{A Y} P_{A Z}$ and $P_{0} P_{A X}$. On $P_{0} P_{A X}, \boldsymbol{q}^{z x} \cdot \nabla \gamma=0$, so that $\gamma$ is constant.

Similarly, define directions on $\pi_{S}$ as $\boldsymbol{p}^{x y} \equiv\left(-S_{x}^{2}, S_{y}^{2}, 0\right)$ $\boldsymbol{p}^{y z} \equiv\left(0,-S_{y}^{2}, S_{z}^{2}\right)$ and $\boldsymbol{p}^{x z} \equiv\left(-S_{x}^{2}, 0, S_{z}^{2}\right)$. Let $\pi_{S V}$ be the intersection of $\pi_{S}$ and $W$. On $\pi_{S V}$, we have $\boldsymbol{p}^{x y} \cdot \boldsymbol{\nabla} \gamma=\left(\gamma+B_{z}\right)\left(\gamma-\gamma_{m}\right)\left(S_{y}^{2}-S_{x}^{2}\right) / \kappa, \boldsymbol{p}^{x z} \cdot \boldsymbol{\nabla} \gamma=$ $\left(\gamma+B_{y}\right)\left(\gamma-\gamma_{m}\right)\left(S_{z}^{2}-S_{x}^{2}\right) / \kappa$, and $\boldsymbol{p}^{y z} \cdot \nabla \gamma=\left(\gamma+B_{x}\right)(\gamma-$ $\left.\gamma_{m}\right)\left(S_{z}^{2}-S_{y}^{2}\right) / \kappa$. When $B_{x}<0, \gamma\left(P_{A Y}\right)=\gamma\left(P_{A Z}\right)=$ $\left|B_{x}\right|$ and (5) implies that $\gamma=\left|B_{x}\right|$ on $Y Z$-plane, and $\gamma+B_{x}>0$ for $X>0$. Since we have seen that $\gamma>\gamma_{m}$ on the vertices on $\pi_{S V}, \boldsymbol{p}^{x z} \cdot \nabla \gamma>0$ and $\boldsymbol{p}^{x y} \cdot \nabla \gamma>0$ everywhere on $\pi_{S V}$, and $\boldsymbol{p}^{y z} \cdot \nabla \gamma>0$ on $\pi_{S V}$ except for segment $P_{S Y} P_{S Z}$.

Now we are in a position to find the maximum of $\gamma$. We must consider the following four cases separately (see Fig. 11).

i) $A_{x} A_{y} \geq S_{z}^{2}$ and $A_{y} A_{z}>S_{x}^{2}$. In this case, $A_{z}>$ $2-A_{0}$ is necessary. $\gamma$ takes its maximum on $P_{S Z}$ and the value is given by (7) and $\gamma<2-A_{0} . \gamma$ approaches 2 only in the limit of $A_{0} \rightarrow 0, A_{z} \rightarrow 2, B_{x} \rightarrow 2$, and $B_{y} \rightarrow 2$. This limit can be taken only if $N \geq 6$, since $A_{y} \geq A_{z}$ must hold in the limit. When $N>6, P_{S Z}$ is the only point that attains $\gamma=2$ since $\boldsymbol{p}^{y z} \cdot \nabla \gamma>0$ and $\boldsymbol{p}^{x z} \cdot \nabla \gamma>0$ still hold in the limit. When $N=6, \gamma=2$ everywhere on $\pi_{A}$, but these states are equivalent in the sense that they are related by the rotation of the whole system.

ii) $A_{x} A_{y}<S_{z}^{2}$ and $A_{y} A_{z} \leq S_{x}^{2}$. In this case, from the relation $0 \leq 4\left(S_{x}^{2}-A_{y} A_{z}\right)=-\left(B_{y}+A_{0}-2\right)\left(B_{y}+\right.$ $\left.A_{0}\right)-\left(B_{z}-B_{y}\right)\left(B_{y}+A_{0}-1\right), B_{y}<2-A_{0}$ is necessary. $\gamma$ takes its maximum on $P_{0} P_{A X}$ and the value is $\gamma=B_{y}<2-A_{0} . \gamma$ approaches 2 only in the limit of $A_{0} \rightarrow 0, A_{x} \rightarrow 2, B_{y} \rightarrow 2$, and $B_{z} \rightarrow 2$. This limit can be taken only if $N \leq 6$, since $A_{x} \geq A_{y}$ must hold in the limit. When $N<6, P_{0}$ coincides with $P_{A X}$ in the limit and $P_{A X}$ is thus the only point that attains $\gamma=2$. When $N=6$, the limit is the same as in the case i).

iii) $A_{x} A_{y} \geq S_{z}^{2}$ and $A_{y} A_{z} \leq S_{x}^{2}$. The maximum is the larger of $\gamma\left(P_{A X}\right)$ and $\gamma\left(P_{S Z}\right)$. Depending on $N$, one of them or both can approach 2 . The limit is the same as described in the cases i) and ii).

iv) $A_{x} A_{y}<S_{z}^{2}$ and $A_{y} A_{z}>S_{x}^{2}$. In this case, from the relation $C\left(P_{A X}\right)>C\left(P_{S X}\right)$, we have $B_{y}>\gamma_{m}$. We also have $A_{0}<2$ since $\left(2-A_{0}\right)\left(B_{y}+A_{0}+2 A_{z}\right)=$ $4\left(S_{z}^{2}-A_{x} A_{y}\right)+2 A_{z}+B_{y}\left(N-2+4 S_{x}^{2}\right) /(N-1)>0$. $\gamma$ takes its maximum at $P_{1}$, that is the intersection of $P_{A Z} P_{A X}$ and $P_{S Z} P_{S X}$. Since $\lambda_{3}=0$ at $P_{1}, \gamma=\lambda_{1}-\lambda_{2}$ and $\beta=\lambda_{1}+\lambda_{2}$. Then we have $\beta^{2}+\gamma^{2}=2 f_{0}$ and $\beta^{2} \gamma^{2}=f_{B}$. This implies that $\gamma^{2}$ is the smaller of the two roots $t=t_{\alpha}, t_{\beta}$ of the equation $t^{2}-2 f_{0} t+f_{B}=0$. The coordinates of $P_{1}$ can explicitly be obtained by solving $f_{A}=0$ and $f_{S}=0$ with $Y=0$. Substituting the result into $f_{0}$ and $f_{B}$ in the equation of $t$, we finally obtain $t_{\alpha}=B_{y}^{2}$ and

$$
\begin{aligned}
t_{\beta} & =\frac{4 A_{x} A_{z}}{\left(B_{y}-\gamma_{m}\right)(N-1)}+A_{0}\left(A_{0}-2\right) \\
& =\left(2-A_{0}\right)^{2}+2 A_{0} \frac{B_{y}+A_{0}-2}{B_{y}-\gamma_{m}}-\frac{2\left(B_{y}+A_{0}-2\right)\left(B_{y}+N\right)+\left(B_{z}-B_{y}\right)\left(B_{y}-B_{x}\right)}{(N-1)\left(B_{y}-\gamma_{m}\right)} .
\end{aligned}
$$

When $B_{y} \geq 2-A_{0}$, we have $t_{\beta}<4-A_{0}\left(2-A_{0}\right)<4$. We thus conclude that $\gamma<2$ for all values of $B_{y}$. When $N<6, \gamma$ approaches 2 only in the limit of $A_{0} \rightarrow 0$, $B_{y} \rightarrow 2, B_{z} \rightarrow 2$, and $A_{x} \rightarrow 2$. $P_{1}$ approaches $P_{A X}$ in this limit, so the limit is the same as in the case ii). When $N>6, \gamma \rightarrow 2$ only in the limit of $A_{0} \rightarrow 0, B_{y} \rightarrow 2$,
$B_{x} \rightarrow 2, A_{z} \rightarrow 2$, and $P_{1} \rightarrow P_{S Z}$. This is the same limit as in the case i). When $N=6, \gamma \rightarrow 2$ in the limit of $A_{0} \rightarrow 0$ and all $A_{\mu}$ approaching 2 .

Finally, we show that $A_{0}-\beta$ is smaller than 2 . In $W, f_{0}$ takes its minimum on $P_{A Z}$, and its value is $\left(A_{x}-A_{y}\right)^{2}+A_{z}^{2}$. We thus have $A_{0}-\beta \leq A_{0}-\sqrt{f_{0}} \leq$ 
$A_{0}-A_{z}=\left(N-2 S_{x}^{2}-2 S_{y}^{2}\right) /(N-1)<N /(N-1)<2$ for $N \geq 3$.

Combining all cases, we reach a conclusion that the maximum value of the concurrence is $2 / N$, and this value is reached only by the state satisfying the constraints $\left\langle\hat{S}_{z}^{2}\right\rangle=(N / 2-1)^{2},\left\langle\hat{S}_{z}\right\rangle=N / 2-1,\left\langle\hat{S}_{x}^{2}\right\rangle=\left\langle\hat{S}_{y}^{2}\right\rangle=$ $(3 N-2) / 4$, and $\left\langle\hat{S}_{x}\right\rangle=\left\langle\hat{S}_{y}\right\rangle=0$, if $z$-axis is suitably chosen. Such a state exists - it is the eigenstate of $\hat{S}_{z}$ with eigenvalue $N / 2-1$ with total spin $N / 2$. This state is equally-weighted in-phase superposition of any one qubit being in the state $|0\rangle$ and the other $N-1$ qubits in the state $|1\rangle$. This is a permutationally invariant pure state that highly (in the order of $N$ ) breaks the symmetry between the basis states $|0\rangle$ and $|1\rangle$.

It is worth noting that the optimal state for our problem was found to be a pure state. This is not trivial because the symmetry is required for density operators. Obviously, possible mixed states span a larger Hilbert space than that spanned by possible pure states.

For entangled chains, the best state so far is a mixed state [17]. It will be interesting to investigate entangled chains composed of a finite number of qubits. Specifically, instead of an infinite line of qubits one can consider an entangled loop composed of even number of qubits $2 N$ (here we assume $N>1$ because for $N=1$ we would have a two-qubit "loop" which is maximally entangled when prepared in a Bell state, in this case the concurrence is equal to unity). One can find a pure $2 \mathrm{~N}$ qubit rotationally-invariant state for which the closestneighbor bi-partite entanglement has the concurrence $C=\left(2+2^{N-2}\right) /\left(2+2^{N}\right)$. In the limit $N \rightarrow \infty$ this concurrence is $C_{\infty}=1 / 4$. We conjecture that this is the maximal value of the concurrence for pure infinite entangled chains. This means that impure states in the limit of infinite number of particles may attain higher bi-partite entanglement than pure states.

This work was supported by a Grant-in-Aid for Encouragement of Young Scientists (Grant No. 12740243) by Japan Society of the Promotion of Science, and by the IST project EQUIP under the contract IST-1999-11053.

[1] E. Schrödinger, Naturwissenschaften 23, 807, (1935); ibid. 23, 823 (1935); ibid. 23, 844 (1935).

[2] A. Einstein, B. Podolsky, and N. Rosen, Phys. Rev. A 47, 777 (1935); J.S.Bell, Physics 1, 195 (1964); A. Peres, Quantum Theory: Concepts and Methods (Kluwer, Dordrecht, 1993).
[3] J. Gruska, Quantum Computing (McGraw-Hill,1999); J. Preskill, Quantum Theory Information and Computation (ww. theory.caltech.edu/people/preskill).

[4] C. H. Bennett, et al., Phys. Rev. Lett. 70, 1895 (1993).

[5] C. H. Bennett and S. Wiesner, Phys. Rev. Lett. 69, 2881 (1992).

[6] A. K. Ekert, Phys. Rev. Lett. 67, 661 (1991).

[7] M. Hillery, et al., Phys. Rev. A 59, 1829 (1999).

[8] C. H. Bennett, et al., Phys. Rev. A 53, 2046 (1996).

[9] V. Vedral, et al., Phys. Rev. Lett. 78, 2275 (1997); V. Vedral, et al., Phys. Rev. A 56, 4452 (1997); V. Vedral and M. B. Plenio, Phys. Rev. A 57, 1619 (1998).

[10] C. H. Bennett, et al., Phys. Rev. A 54, 3824 (1996).

[11] S. Hill and W. K. Wootters, Phys. Rev. Lett. 78, 5022 (1997); W. K. Wootters, Phys. Rev. Lett. 80, 2245 (1998).

[12] M. Horodecki, et al., Phys. Rev. Lett. 84, 2014 (2000).

[13] B. Kraus, et al., arXiv quant-ph/9912010 (1999); P. Rungta et al. arXiv quant-ph/0001075 (2000).

[14] A. V. Thapliyal, Phys. Rev. A 59, 3336 (1999); J. Kempe, Phys. Rev. A 60, 910 (1999).

[15] D. M. Greenberger, et al., Am. J. Phys. 58, 1131 (1990).

[16] V. Coffman, et al., arXiv quant-ph/9907047 (1999).

[17] W. K. Wootters, arXiv quant-ph/0001114 (2000).

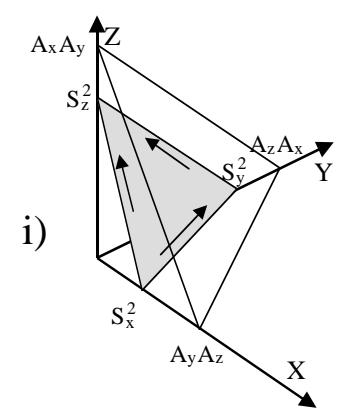

ii)
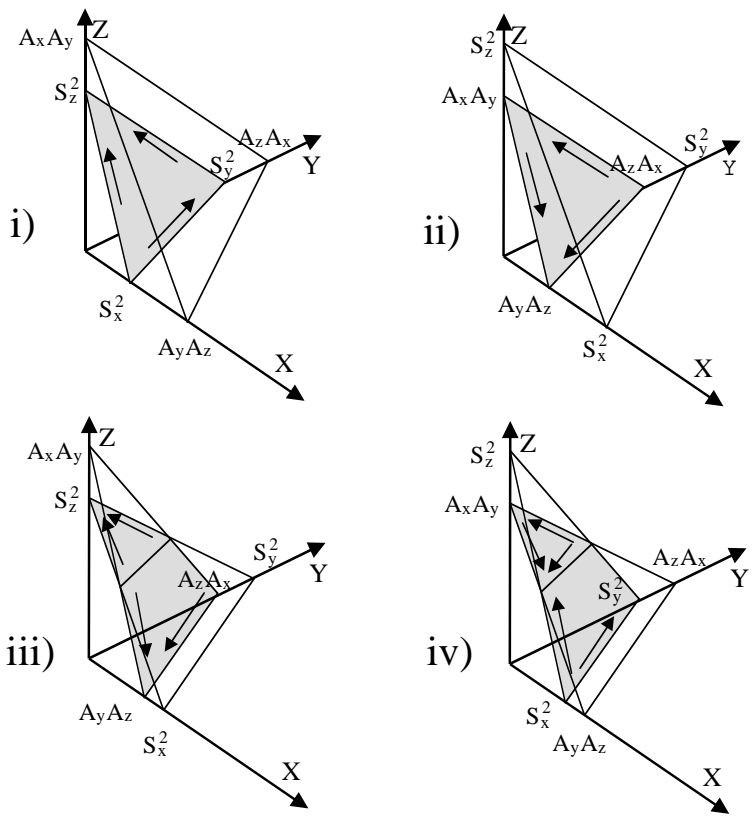

FIG. 1. Allowed regions for the parameters $\{X, Y, Z\}$. The shaded surfaces are boundaries for the physically allowed states. The arrows show the directions in which the concurrence increases. 